\title{
Palpebral Conjunctiva
}

National Cancer Institute

\section{Source}

National Cancer Institute. Palpebral Conjunctiva. NCI Thesaurus. Code C12901.

The part of the conjunctiva, a thin transparent mucous membrane, that covers the posterior surface of the eyelids. 